Artículo Original

\title{
Aproximación a la performance como estrategia política de resistencia al apartheid ocupacional: la situación de las personas travestis
}

\author{
Aproximação à performance como estratégia politica de resistência ao \\ apartheid ocupacional: a situação das pessoas travestis
}

\section{Approach to the performance as a political strategy of resistance to occupational apartheid: the situation of transvestite people}

\author{
Mariana Fuentes-Barahona ${ }^{a}$ (D), Camila Lara-Saldaña ${ }^{a}$ (D), Marianne Pfeifer-Fuentes ${ }^{a}$ (D), \\ Sofía Zapata-Carrasco ${ }^{a}$ (D), Débora Grandón-Valenzuela ${ }^{b}$ (D), Rodolfo Morrison ${ }^{a}$ (D)

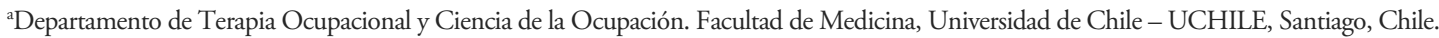 \\ bPrograma de Doctorado en Estudios Latinoamericanos, Centro de Estudios Culturales Latinoamericanos, Universidad de Chile - UCHILE, \\ Santiago, Chile.
}

Cómo citar: Fuentes-Barahona, M., Lara-Saldaña, C., Pfeifer-Fuentes, M., Zapata-Carrasco, S., GrandónValenzuela, D., \& Morrison, R. (2021). Aproximación a la performance como estrategia política de resistencia al apartheid ocupacional: la situación de las personas travestis. Cadernos Brasileiros de Terapia Ocupacional, 2021, e3127. https://doi.org/10.1590/2526-8910.ctoAO23953127

\begin{abstract}
$\underline{\text { Resumen }}$
Introducción: Las personas travestis se ven enfrentadas a diferentes situaciones de injusticias sostenidas por un sistema heteronormado que violenta sus vidas, corporalidades y silencia sus identidades. En este sentido, el apartheid ocupacional opera a través de la conformación de una estructura que limita sus posibilidades ocupacionales, pero, al mismo tiempo, las personas travestis podrían poner resistencia a ese ejercicio del poder a través de diferentes mecanismos como las performances. Objetivo: Explorar y analizar la expresión artística y política de la performance, realizada por disidentes sexuales, como estrategia política de resistencia al apartheid ocupacional. Metodo: Este estudio exploratorio corresponde a un diseño de estudio de caso, basado en el análisis documental audiovisual. Se analizó una pieza audiovisual denominada "Yo me maquillo" performada por dos artistas y activistas disidentes sexuales en la ciudad de Valparaíso (Chile). Para el análisis, se realiza una codificación abierta de elementos significativos y una codificación axial posterior. Resultados: Se evidencia cómo la performance opera como una estrategia política de resistencia al apartheid ocupacional que personas travestis experimentan en sus vidas cotidianas, a través de la denuncia, visibilización, acción, agitación y desafío de las matrices hegemónicas, permitiendo dar cuenta de las desigualdades y violencias
\end{abstract}


estructurales que cruzan sus vidas. Conclusiones: Se discuten los aportes del estudio para la terapia ocupacional y la ciencia de la ocupación. Se destaca la performance como una ocupación colectiva y política que se desarrolla desde la resistencia y subversión al apartheid ocupacional que viven las personas travestis.

Palabras-clave: Arte, Política, Estudios de Género, Terapia Ocupacional.

\section{Resumo}

Introduçáo: As travestis se deparam com diversas situaçôes de injustiça sustentadas por um sistema heteronormado que viola suas vidas, sua corporeidade e silencia suas identidades. Nesse sentido, o apartheid ocupacional opera por meio da formaçáo de uma estrutura que limita suas possibilidades ocupacionais, mas, ao mesmo tempo, as travestis poderiam resistir a esse exercício de poder por meio de diferentes mecanismos, como as performances. Objetivo: Explorar e analisar a expressão artística e política da performance, realizada por dissidentes sexuais, como estratégia política de resistência ao apartheid ocupacional. Método: este estudo exploratório corresponde a um desenho de estudo de caso, baseado na análise documental audiovisual. Foi analisada uma peça audiovisual intitulada "Yo me maquillo" [Eu me maquio], interpretada por dois artistas chilenos e ativistas dissidentes sexuais na cidade de Valparaíso (Chile). Para a análise, é realizada uma codificação aberta dos elementos significativos e uma codificação axial posterior. Resultados: Evidencia-se como a performance opera como estratégia política de resistência ao apartheid ocupacional que as travestis vivenciam em seu cotidiano, por meio da denúncia, visibilidade, ação, agitação e desafio das matrizes hegemônicas, permitindo dar conta das desigualdades e violências estruturais que cruzam suas vidas. Conclusóes: São discutidas as contribuiçóes do estudo para a terapia ocupacional e a ciência ocupacional. A performance é destacada como uma ocupaçáo coletiva e política que se desenvolve desde a resistência e subversão ao apartheid ocupacional vivido pelas travestis.

Palavras-chave: Arte, Política, Estudos de Gênero, Terapia Ocupacional.

\section{$\underline{\text { Abstract }}$}

Introduction: Transvestite people face different situations of injustice sustained by a heteronormative system that violates their lives, bodies, and silences their identities. In this sense, Occupational Apartheid operates through the formation of a structure that limits their occupational possibilities, but, at the same time, transvestite people resist this exercise of power through different mechanisms such as performances. Objective: To explore and analyze the artistic and political expression of performance, developed by sexual dissidents, as a political strategy of resistance to Occupational Apartheid. Method: This exploratory study corresponds to a case study design, based on audiovisual documentary analysis. An audiovisual piece called "Yo me maquillo" [I put on makeup] performed by two Chilean sexual dissidents and activists was analyzed in the city of Valparaíso (Chile). For the analysis, open coding of significant elements and posterior axial coding was performed. Results: It is evident how the performance as a political strategy of resistance to the occupational apartheid experienced by transvestite people in their daily lives, through the denunciation, visibility, action, agitation, and defying the hegemonic matrices, allowing to account for inequalities and structural violence that crosses their lives. Conclusions: The contributions of the study for occupational therapy and occupation science are discussed. Performance is highlighted as a collective and political occupation that develops from the resistance and subversion to occupational apartheid experienced by transvestite people.

Keywords: Art, Politics, Gender Studies, Occupational Therapy. 


\section{Introducción $^{1}$}

Como grupo de mujeres cis-género disidentes sexuales buscamos introducirnos en la discusión sobre las problemáticas que afectan la vida de las personas travestis, quienes son excluidas, violentadas y vulneradas sistemáticamente en función de las normas de sexo, género y deseo, siguiendo las discusiones disciplinares sobre la relación de la terapia ocupacional con la diversidad sexual (Aguiar et al., 2020; Braga et al., 2020; Gómez-Antilef et al., 2020; Melo, 2016; Monzeli, 2013; Monzeli et al., 2015; Morrison et al., 2020).

Estas situaciones de violencia se han manifestado de manera estructural en cuestiones como la exclusión al acceso a salud, trabajo, educación, y seguridad personal y social, además de crímenes de odio, hostigamientos sexuales, agresiones, maltrato físico y psicológico y discriminación en distintos aspectos y lugares donde desarrollan su vida (Buriticá, 2013), siendo la comunidad travesti un grupo altamente vulnerado y expuesto a situaciones de violencias que, en un alto porcentaje, culminan en su muerte (Radi \& SardáChandiramani, 2016).

Según el Informe de Abusos y Violaciones de los Derechos Humanos, las vulneraciones de los derechos de las personas travestis no encuentran justicia, y de hecho, un gran número de las denuncias realizadas no son investigadas, quedando sin responsables y en completa impunidad (Centro de Documentación y Situación Trans en Latinoamérica y el Caribe, 2017).

Dicha situación de exclusión y vulneración sistemática puede ser analizada desde conceptos como el Apartheid Ocupacional (en adelante, AO) (Kronenberg \& Pollard, 2006) lo que explica en gran medida la restricción al ejercicio pleno de derechos humanos por la comunidad travesti, que en países como Brasil y Argentina se expresa en que mayoritariamente las personas travestis deban desempenarse principalmente en la prostitución, como única posibilidad laboral disponible (Bonassi et al., 2015).

Brasil es el país donde ocurren más asesinatos de travestis y transexuales. En términos absolutos, el número de homicidios es casi cuatro veces más grande que México, que ocupa el segundo lugar en el ranking. Además, las personas travestis presentan dificultades de acceso a servicios comunitarios e institucionales atravesadas en tres dimensiones, una individual (expresada por valores, actitudes y relaciones), una social (desde referentes culturales, transgeneracionalidad, empleo, apoyo social, ocio) y lo programático (como políticas públicas, gobernabilidad, acceso a servicios, etc.) lo que constituye limitantes de promoción de su salud, pues a nivel latinoamericano existen pocos programas que se centren de forma particular en esta población (Gutierres \& Lordello, 2020).

Si bien Argentina cuenta con la Ley de Identidad de Género, una de las más avanzadas a nivel mundial, pues despatologiza las identidad trans/travestis y permite el acceso gratuito a cambios registrales y a atenciones en salud, las personas travestis aún se enfrentan situaciones como:

[...] violencia institucional y social -los crímenes de odio, persecución policial-; acceso precario a la salud -la realización de prácticas de inyección de siliconas industrial no supervisadas por profesionales; el VIH/sida; la fuerte exclusión del mercado laboral [...] y la discriminación, maltrato social y familiar -que ante la

\footnotetext{
${ }^{1}$ Para fines de este escrito se escribirá desde el lenguaje no binario, utilizando la letra "e" en pronombres y sufijos (Castillo \& Mayo, 2019).
} 
falta de contención se generan situaciones de angustias graves, depresión y en muchos casos suicidios (Boy \& Rodríguez, 2019, p. 2).

En Chile, la situación de vulneración y discriminación no difiere de estos países, dificultándose el acceso de este grupo hacia múltiples derechos sociales, además de existir situaciones de amedrentamiento, que son ejercidas por grupos como los Neo-nazi, quienes violentan de forma directa el derecho a la vida, a la libertad y a la seguridad personal (Instituto Nacional de Derechos Humanos, 2012). En este sentido, el caso argentino es el más destacable en términos legislativos, pues cuentan con la Ley Nacional 26.743 sobre "Identidad de Género", que reconocer el ser persona travesti como un derecho protegido (Argentina, 2014).

El concepto de $\mathrm{AO}$ apareció en la disciplina ante la falta de términos que conceptualizasen las injusticias sistemáticas y estructurales que viven personas y grupos en situaciones de vulneración, y que son responsables de negarles el acceso a la participación en ocupaciones significativas (Kronenberg \& Pollard, 2006). Es por lo anterior, que puede observarse una experiencia de apartheid ocupacional en base al género en la vida de las personas travestis como, puesto que:

[...] la segregación de grupos de personas mediante la restricción o negación de su acceso a una participación digna y significativa en las ocupaciones de la vida diaria, basada en la raza, color, discapacidad, procedencia nacional, edad, sexo, orientación sexual, religión, creencias políticas, u otras características. Ocasionado por fuerzas políticas, sus consecuencias sociales, culturales y económicas sistemáticas y dominantes, ponen en peligro la salud y el bienestar de individuos, comunidades y sociedades (Kronenberg \& Pollard, 2006, p. 66).

Acorde con lo planteado por Pollard et al. (2008), podemos plantear que las personas travestis viven una restricción por parte de estructuras y grupos con mayor poder por el desprecio de sus derechos humanos esenciales, lo que reproduce una ciudadanía de primer orden para personas cisgénero y una ciudadanía negada, marginal o de segundo orden, para las personas travestis y trans (Moraga, 2017). En este sentido, además de ser objeto de violencia física, verbal y psicológica, estas no son reconocidas por su identidad, encontrándose en una desprotección casi total por parte del Estado y sus instituciones, las que a su vez, generan también violencias, exclusión, discriminación, y barreras de acceso al trabajo (García, 2010). Además de violentada, la comunidad travesti ha sido limitada en sus elecciones ocupacionales, lo que se observa de forma patente en la limitación de posibilidades laborales que someten a las personas travesti a desempeñarse casi exclusivamente en el trabajo sexual inseguro, por el cual además resultan en muchas ocasiones, privadas de libertad (Torres, 2013).

En Chile, para la terapia ocupacional (TO) y la ciencia de la ocupación (CO), aún existe una escasa vinculación con la comunidad de personas travestis, fenómeno que si bien obstaculiza el acceso a estudios previos e información sobre la temática, permite comprender la gran relevancia de profundizar las investigaciones en esta área. En este sentido, existen escritos que han abordado las diferentes problemáticas que enfrentan personas no heterosexuales como el impacto del AO en parejas del mismo sexo (Morrison et al., 2020), o como la heterosexualidad normativa restringe las elecciones ocupacionales de personas no heterosexuales (Aguiar et al., 2020; Avillo et al., 2015), o la necesidad de promover la justicia ocupacional y el reconocimiento de derechos, evitando los impactos en salud mental y calidad de vida (Marchant Castilho, 2019; Morrison et al., 2019). 
Para ambas disciplinas ha sido de interés reciente abordar la experiencia cotidiana de las diversidades sexo-genéricas, comprendiendo los escenarios de violencia e injusticia social en el que éstas se encuentran inmersas; pero también, las formas de agencia que se despliegan desde este sector, con una mirada política y de transformación. En este sentido, las producciones artísticas de la comunidad travesti chilena cuentan con una larga data y desarrollo, con el trabajo de referentes como Carlos Leppe, Pedro Lemebel, las Yeguas del Apocalipsis, Hija de Perra, Giuseppe Campuzano, Francisco Copello, entre otras. Dentro de estas producciones artísticas destacan las performances, que buscan desestabilizar las cotidianidades mediante acciones de arte y que permiten poner en escena aquellos cuerpos disidentes, silenciados y violentados (Cellino, 2015). Para Cortés et al. (2016) el arte performativo es una obra desarrollada por un individuo o grupo, que cuenta con cuatro elementos básicos: "tiempo, espacio, el cuerpo del/los artistas y una relación entre estos y el público" Cortés et al. (2016, p. 11). De esta manera, cada performance implica diferencias que radican en los contextos de producción así como el discurso de cada artista, dando vida a una amplia gama de posibilidades, siempre tras un deseo militante de expresión (Gómez, 2013).

Considerando el rol social y crítico de la $\mathrm{TO}$ y la $\mathrm{CO}$, la presente investigación pretende explorar si las performances de las personas travestis actúan como estrategias políticas frente a la situación de $\mathrm{AO}$ que cruza sus cotidianidades, comprender cuáles son las expresiones o señales de $\mathrm{AO}$ y cómo se responde a éstos a través de la performance. Lo anterior, con el fin de contribuir desde la dimensión política, ética y situada que configura a la disciplina, hacia la visibilización, respeto y validación de la identidad travesti, analizando críticamente sus acciones, experiencias y luchas. Para lo anterior, se analiza una performance realizada en la ciudad de Valparaíso (Chile) por dos activistas de Chile.

\section{La identidad Travesti como una Fuga de la Matriz Heterosexual y Binaria del Género}

El género se puede comprender y referir desde distintas perspectivas. Scott (1996) lo entiende como una categoría vinculada al poder, distinguiendo que tanto el sexo como el género son elementos constitutivos de las relaciones sociales, lo que corresponde a una forma primaria de las relaciones de poder. Esta noción fue criticada por omitir las otras relaciones de poder que se circunscriben sobre los cuerpos, como la etnia, clase, o experiencias de dominación colonial (Tarrés, 2013). Al respecto, Barbieri (1993) aborda el género desde las desigualdades sociales, incluyendo dentro de estas, aspectos del contexto de tipo étnico-culturales y del ciclo vital de las personas. Marta Lamas profundiza lo anterior al señalar que "[...] el género es una lógica cultural omnipresente en todas las situaciones sociales, que tiñe la construcción de la identidad psíquica y que condiciona el sistema jurídico" (Lamas, 2017, 33m57s). Por otro lado, Butler (2007) refiere que el género no se concibe como una inscripción cultural sobre un sexo biológico preexistente, pues el cuerpo sería aquel que se produciría o materializaría a través del discurso, sin existir una distinción entre sexo y género como tal.

Para la filósofa estadounidense, dicha construcción se encuentra enmarcada dentro de la matriz heterosexual (Butler, 2007), la cual apunta a la imposición de la heterosexualidad y del sistema binario como norma, que define al mundo a través de dicotomías regidoras, en este caso hombre/mujer, heterosexual/homosexual, normal/anormal, blanco/negro, viable/no viable, etc. (Duque, 2010). Esto, produce una categorización binaria del sexo, que origina a 
su vez, una correlación lógica entre sexo, género y deseo, en donde el primero se sitúa en la base, como causa de una experiencia, conducta y deseo sexual específicos, diferenciando lo humano de lo inhumano en base a una legitimidad socialmente atribuida (Butler, 2007).

Así, lo humano sería aquello que posee la cualidad de ser inteligible, por encima y en contra de lo inhumano, que serían todas aquellas expresiones que se excluyen del binomio hombre-mujer. Asimismo, cuando las identidades no se expresan según esta concatenación causal, se constituyen identidades no viables para la normativa y que, por tanto, son coartadas al momento de respirar, amar y vivir (Butler, 2007).

En este sentido, la teoría de performatividad del género de Butler $(2002,2007)$ explica que el género se construye a través de la repetición y ritual constante de los actos, impulsados y sostenidos por las restricciones normativas existentes, y que se encarna en la estilización del cuerpo. Asimismo, las normas de género otorgan una pauta de acción, en las que el género forma 'parte del hacer', que si bien le permite ser actuado por las personas, no es un mandato fijo (Polanco \& Morrison, 2019). De forma paralela, las identidades se configuran por medio de ocupaciones y en el proceso de ocuparnos: las personas se construyen en la medida en que dotan de sentido y significado las actividades que realizan (Guajardo, 2012). Es en dicho "hacer" que se va definiendo una identidad ocupacional "individual" que es, a su vez, expresión de la colectiva, en donde las ocupaciones "expresan los gestos, los movimientos y las acciones concretas que las personas desempeñan para construir su género" (Polanco \& Morrison, 2019, p. 79). De esta manera, se entiende que mediante los actos performativos se hace el género, y estos actos al mismo tiempo, se constituyen por medio de la ocupación.

Así, la performatividad no trata simplemente de una repetición de actos, inherentemente intactos e idénticos, sino que también, es en esta reiteración que se tiene la posibilidad de desestabilizar estas normativas (Butler, 2002) y es a través de estas acciones que se manifiestan aquellas identidades que no encajan en las normas de inteligibilidad cultural, dando la oportunidad de mostrar los límites y los propósitos reguladores de esta, para así, dar a conocer otras matrices subversivas de desorden de género (Butler, 2007). Lo anterior, se vuelve importante en la lucha por el cuestionamiento de los ideales corporales o identitarios, posibilitando un futuro en que se permita la valoración de las distintas personas, vidas, identidades, cuerpos, materialidades, etc. (Butler, 2004). En este sentido, destaca la importancia de entender lo queer ${ }^{2}$ como algo que rebasa lo puramente identitario, puesto que debe ser entendido como un concepto a "[...] aplicar cuando establecemos alianzas incómodas o impredecibles en la lucha por la justicia social, política y económica" (Butler, 2017, p. 75).

Dentro de las expresiones queer latinoamericanas, lo travesti, como concepto, tiene muchos significados e interpretaciones, que incluyen su uso como una ofensa, un concepto despreciativo y de odio (Gómez, 2013), pero también existen otras que se relacionan con una identidad política, de reivindicación, de resistencia al binarismo y toda la opresión que representa (Kulawik, 2008). Si bien, el concepto ha sido ampliamente desarrollado en países de la región latinoamericana, en la sociedad chilena aún se tipifica dentro de un espectro “Trans” (Instituto Nacional de Derechos Humanos, 2012).

Se puede observar que las conceptualizaciones en torno a las personas travestis no son realmente representativas y responden así a otra forma de invisibilización, homogeneización e higienización de las identidades sexo-genéricas, pasando por alto su trayectoria y sentido político, y reforzando los prejuicios que las posicionan en un nivel inferior o exclusivamente

\footnotetext{
${ }^{2}$ Lo queer expresa el concepto de desequilibrar o perturbar; por lo que las prácticas queer se apoyan en la noción de desestabilizar normas que están aparentemente fijas (Fonseca \& Quintero, 2009).
} 
ligadas al trabajo sexual (Aguiar et al., 2020). En esta línea, Pierce (2020, p. 307) señala que el término transgénero "[...] despolitiza una historia violenta de marginación social y económica. El término travesti, por el contrario, conserva esta diferencia de clase y resonancia popular y, por lo tanto, es una identificación política, más que psicológica o incluso corporal".

De esta forma, esta identificación se establece como un acto de memoria de lucha, de posición de clase y género contra-heteronormada, disidente, y de resistencia, configurándose como una expresión de inconformismo y oposición (Kulawik, 2008). Las concepciones de lo travesti transitan en lo ambiguo, proponiendo un desborde de las nociones tradicionales de lo femenino y lo masculino desde una perspectiva del otro, del marginado, sancionado y censurado por el sistema sexo-género y su artificiosa normalidad lineal de deseos y representaciones corporales (Kulawik, 2008).

Se desprende así esta naturaleza performativa del travestismo (Maureira, 2009) la cual se refiere a la representación exagerada de la norma, pero que no trata de copiarla para aparentar pertenecer a ella, o transformar el cuerpo hasta mimetizarse, sino de transgredir los límites que este modelo impone, desnudarlo y hacer visibles sus falencias y atributos inestables (Kulawik, 2008). Claudia Rodríguez, reconocida artista y activista travesti, plantea que el ser travesti es romper con todo lo que se quiera, ya sea la familia, el cuerpo, el amor o cualquier naturaleza, destacando que una persona travesti tiene derecho a ser monstruo (Presentes LGTB, 2017). Así, para Rodríguez (en Carrasco, 2015) lo travesti sería una realidad biográfica cruzada por la precariedad, subalternidad y la violencia, donde su existencia constituye un grito de las eternamente excluidas, incluso en los espacios de diversidad y disidencia sexual, en donde "[...] monstruosiarse se ha convertido en una salida para cuestionar cómo se entiende el cuerpo en relación con los mecanismos de control biopolítico [...] Monstruoar (como verbo) representa un modo crucialmente queer de resistencia a la normatividad epistemológica" (Pierce, 2020, p. 308). Dado que las personas travestis desafían la heterosexualidad obligatoria y el sistema sexo-género binario, su existencia representa una ruptura y desestabilización del sujeto masculino heterosexual que es representado por la cultura hegemónica (Kulawik, 2008). Así, a medida que se perturba el orden establecido por los cánones de género y clase, y su respectiva normatividad, el cuerpo travesti se convierte en un lugar de transgresión (Buriticá, 2013).

\section{El Carácter Político de las Ocupaciones y la Cotidianidad}

Dentro de la matriz heterosexual y binaria existe un espacio que permite subvertir la norma, y bajo este escenario surgen las estrategias políticas como una posibilidad de subvertir prácticas de opresión, especialmente aquellas determinadas por la matriz heterosexual (Vidiella, 2014). A partir de esta posibilidad, se alzan desde los sectores disidentes, estrategias de acción y organización, las que Neer (2017) denomina como organizaciones sexo-politicas. Estas tienen el fin de denunciar y contraponerse a la discriminación, invisibilización, estigmatización, desigualdad u otras formas de exclusión normativas, que las personas travestis viven a lo largo de su cotidianidad.

Así, se da pie a procesos de construcción de subjetividades que permitan la creación de micropolíticas emancipatorias, de transformación social y producción de sujetes autónomes, y desde ahí construir un sistema propio de referencias independiente de la normatividad rigente. Se configuran entonces, estrategias que recorren matices artísticos e ideológicos, posibilitando hacer patente aquello que parece no existir, y así conformar esferas de 
movilización y encuentro para construir una realidad más democrática (Pérez, 2013). El desarrollo de estas estrategias surge desde el entramado de relaciones sociales y de poder dadas en la vida cotidiana, entendiendo a ésta como un fenómeno amplio y complejo, que es irreductible a contextos específicos o a la realización de acciones diarias, sino que se involucra en los modos de vida de las personas, sus ocupaciones e identidades (Galheigo, 2003).

Lefebvre (1991) posiciona a la vida cotidiana como un escenario donde se expone la totalidad de lo real, lo que implica develar todo aquello que abarca y expone. Por consiguiente, en cada espacio y lugar se configuran cotidianidades distintas, las que están sujetas a las estructuras dominantes. Bajo este supuesto, Galheigo (2020) utiliza la metáfora del tejido como una forma de esquematizar y entender cómo la vida cotidiana contiene y entrelaza las capas y estructuras de la existencia humana, y como un medio para zurcir las micro y macroestructuras que configuran lo cotidiano.

En vista de ello, Galheigo (2003) propone desarrollar una postura crítica ante y desde la vida cotidiana que permita vislumbrar la dominación de los modelos estructurales, así como también, los efectos de ésta en las vidas de las personas, principalmente los sufrimientos e injusticias, desde los cuales surgen anhelos de cambio y transformación del sujeto individual como del entorno. Tal como lo plantean Pardo \& Silva (2015, p. 125) “[...] se trata de develar la naturaleza política de las ocupaciones y los espacios de cotidianidad [...] reconociendo que representan la posibilidad de influenciar en los ámbitos sociales dada la capacidad transformadora de la actividad humana”. Kronenberg \& Pollard (2006) señalan que esta capacidad hace valer el poder que toda ocupación humana posee, entendiendo la relación dinámica y bidireccional que existe entre la ocupación y el entorno global. De ahí que refieren que las actividades politicas se consideran como una ocupación, dado que estas van desde los intereses personales hasta tener un impacto tanto local como global, y se concretan en los medios que tenemos como personas para realizar nuestras ocupaciones diarias (Pollard et al., 2008).

En este sentido, los autores proponen el concepto de Actividades politicas de la Vida Diaria (ApVD), siendo lo político algo que tiene lugar en todas partes y en todos los aspectos de la vida (Kronenberg \& Pollard, 2006). Así, existe una interrelación entre la ocupación y la base de la política que es muy significativa, la cual se evidencia en la capacidad de les individues para participar e incidir en actividades comunitarias en cualquier nivel de la organización social (Pollard et al., 2008).

Dicho esto, la ocupación también está relacionada a la cultura y sus manifestaciones, dado que puede ser considerada "[...] una expresión singular y colectiva, [...] y también una creación sensible, subjetiva, que viabiliza las formas de existir en el mundo” (Silva et al., 2017, p. 107). Dentro de estas se encuentra el arte, tanto su producción y desempeño en alguna de sus formas, como su deleite y apreciación, pues otorga espacios esenciales de expresión, comprensión, creación, existencia de diversidades y participación sociopolítica (Silva et al., 2017), posibilitando espacios de cambio y resignificación de los sentidos. Dadas estas características es que podemos considerar al arte no sólo como ocupación, sino también como estrategia política que podría llegar a agitar las normatividades vigentes. Será desde esta consideración que la presente investigación explorará si las performances de la comunidad travesti pueden considerarse como estrategias de subversión al AO que históricamente ha configurado su cotidianidad.

\section{Metodología}

Esta investigación se ha posicionado desde una epistemología feminista que rechaza la neutralidad y objetividad con pretensiones totalizadoras, y procura eliminar cualquier 
ímpetu sustancializador, planteando la existencia de perspectivas parciales, que siempre producen su saber de una forma situada, como condición epistemológica (Haraway, 1991).

Además, el estudio se ha realizado desde un paradigma y metodología cualitativa desde un diseño exploratorio (Pérez, 1998), cuyos principios orientadores son la comprensión e interpretación de lo que sucede en una situación en concreto. Además, se ha utilizado el método de estudio de caso, el cual, según Chetty, resulta apropiado para analizar en profundidad y desde múltiples perspectivas problemas o situaciones emergentes, dadas en contextos singulares (Martínez, 2006).

La técnica corresponde a un análisis de material audiovisual, que según Pink describe y recopila el trabajo de los materiales audiovisuales con el objetivo de comprender, expresar o explicar fenómenos (Nayar \& Stanley, 2014). El uso de lo visual constituye una herramienta para consolidar la comprensión de experiencias o la representación simbólica de fenómenos sociales y políticos, posicionándolo en un escenario de relaciones comunicacionales horizontal y simétrico, que fortalece la experiencia y perspectiva del actor social (Aguilar, 2016). Dulzaides \& Molina (2004, p. 2) proponen el análisis de información, "[...] cuyo objetivo es la captación, evaluación, selección y síntesis de los mensajes subyacentes en el contenido de los documentos, a partir del análisis de sus significados, a la luz de un problema determinado". Este se ha realizado en primer lugar, mediante una codificación abierta la cual establece códigos preexistentes in vivo (Bonilla \& López, 2016), para luego proceder a una codificación axial, la cual relaciona estas categorías entre ellas y con sus sub-categorías, presentando explicaciones más precisas y completas acerca del objeto de estudio (Valdés, 2016).

Finalmente, para llevar a cabo esta investigación se ha analizado una obra audiovisual realizada el ańo 2020, que corresponde a una performance de artistas disidentes sexuales desarrollada en la ciudad de Valparaíso (Chile), cuyo contenido aborda la experiencia travesti. Se ha escogido como ciudad de producción Valparaíso, dado que ésta constituye la zona con mayor cantidad de denuncias y casos de homofobia y transfobia en Chile de acuerdo al $19^{\circ}$ Informe Anual de Derechos Humanos de la Diversidad Sexual y de Género (Movimiento de Integración y Liberación, 2021), además de ser un territorio que destaca por su carácter artístico y político, en donde han sucedido una gran cantidad de intervenciones performáticas a lo largo de los años.

\section{Análisis}

\section{Descripción y análisis general de la performance}

La performance analizada lleva como nombre "Me Maquillo"3,4 - (Balmánica, 2020), protagonizada por las artistas disidentes sexuales Anna Balmánica5 y Organa Feminazi ${ }^{6}$. Ésta se desarrolla a los pies de la Plaza "El Descanso" ubicada en la ciudad de Valparaíso, en el contexto del primer aniversario de la Revuelta Popular de Octubre del 2019 en Chile. A partir de lo observado en la performance, se definieron categorías de análisis en torno a

\footnotetext{
${ }_{3}^{3}$ Disponible en la cuenta de Instagram de Anna Balmánica (@basmanicx) (Balmánica, 2020).

${ }^{4}$ Se sugiere a les lectores ver la performance antes de continuar leyendo el artículo.

${ }^{5}$ Anna Balmánica es un personaje creado por el diseńador gráfico y transformista Bastián Pérez. Instado por el fallecimiento de Hija de Perra (una importante "artivista”, disidente sexual e ícono en la cultura chilena), Anna Balmánica comenzó a representar al arte del transformismo como un acto político, cuya responsabilidad es empoderar y visibilizar las injusticias y violencias en la comunidad travesti, lésbica y otras (Toro \& Carrasco, 2019).

${ }^{6}$ Organa Feminazi, oriunda de Valparaíso (Chile), es una activista, disidente sexual y estudiante de psicología, que ha expuesto los abusos y maltratos que enfrentan las personas travestis a través de diferentes intervenciones artísticas en espacios públicos (Coñuenao, 2019).
} 
los principales tópicos que estas desarrollan y a los objetivos de esta investigación. Entre estas destacan descripción general de la performance, señales de apartheid ocupacional y señales de subversión al apartheid ocupacional.

Como parte del análisis de la performance, se pudieron determinar al menos tres actos que reproducen una misma escena, pero que va cambiando de énfasis e intencionalidad, repitiendo algunos diálogos, intercalando a la interlocutora, reiterando acciones, etc. A lo largo de la performance las protagonistas encarnan distintas personalidades y facetas, en las que inclusive representan aquellas visiones sociales de odio hacia lo travesti, haciendo parte de sí insultos, violencias, y prejuicios.

La primera escena encarnada por Anna Balmánica, alude a la intencionalidad que guarda el uso del maquillaje. Aquí se manifiesta una visión asociada a un rol "estético", utilizado para sentirse bonita y bien consigo misma y que refiere en sus dichos, "me maquillo para gustarme, me maquillo para sentirme rica, me maquillo para no ser un desastre". Esto último guarda estrecha relación con aquella imagen que exterioriza (dado el uso de prendas y elementos típicamente asociados a lo femenino, como el maquillaje, la lencería y los peinados), la cual está sujeta a la permanente opinión social que materializa las identidades y corporalidades dentro de los preceptos binarios. De forma recurrente Anna pregunta a les espectadores " ¿ustedes me ven fea?", reafirmando este ideario coherente con lo femenino, y expectante frente a la aprobación simbólica de parte del público, cuya respuesta podría dar cabida a juicios y sentencias que impacten sobre sus cuerpos y vidas.

Luego, se observa que lo planteado por Anna se contrasta con lo que exclama Organa en este acto, quien juega un rol más bien antagónico a la protagonista de la escena, encarnando un discurso que se interpreta como la representación de lo que piensa la sociedad, o quienquiera que mira estos cuerpos y genera una opinión, en dichos tales como "ieres fea?" y "puta sidosa", los cuales responden a los actos e ideas sostenidas por las restricciones normativas que llegan a estilizar los cuerpos (Butler, 2002, 2007). Lo anterior, responde a los cánones estéticos y morales que invitan a pensar de forma limitada en aquello que es bueno, bonito y sano dentro de los márgenes de la matriz heterosexual, lo que se expresa, por ejemplo, en asumir la belleza de una forma determinada y única, como una esencia estática que modela a los cuerpos, lo que va a la par de la connotación negativa hacia las personas con VIH/SIDA.

Además de estas frases, Organa refiere que se maquilla por otras razones muy diferentes, como contagiar, intoxicar, arruinar, denunciar, y funar ${ }^{7}$, aspectos que pueden indicar el pensamiento de otres sobre estas existencias travestis, lo que se relaciona con lo planteado sobre las concepciones de ser travesti como personas que se dedican a la prostitución, o son asociadas al trabajo callejero, informal y precario, ideas que incluso pueden llegar a ser internalizadas y reproducidas por estas mismas.

Otro elemento importante fueron los diálogos en donde las protagonistas piden perdón, que son los momentos que demarcan el final de cada acto, y son precedidos por el mismo diálogo cada vez: "me maquillo porque me siento insana, me maquillo porque yo soy la enfermedad'. Esta parte reiterada durante la performance es entendida como una alusión a la concepción de sí mismas y la naturaleza de sus cuerpos disidentes, que llegan como consecuencia de lo que es exclamado por esta sociedad atiborrada de estereotipos gestados dentro de una matriz heterosexual binaria, que además es en gran parte cristiana. Dados

${ }^{7}$ Es un verbo empleado a modo de modismo en Chile que implica realizar una denuncia, ya sea a través de un acto público o en redes sociales, que implica develar actos de represión o injusticias cometidas en momentos pasados por personas o instituciones. 
estos antecedentes, es que se comprende el acto de pedir perdón, sobre todo hacia el final de la performance cuando ambas protagonistas juntan sus manos a modo de rezo y ruegan que las perdonen mirando al cielo, porque se les ha hecho creer que ellas son el problema, quienes traen "la enfermedad", quienes están mal y no pertenecen a este mundo. Lo anterior, puede explicarse en base al rol e influencia de la religión judeocristiana, que instaura modelos del buen actuar y las buenas costumbres, fundamentando así los cimientos de una supuesta "ley natural" que no da cabida a transgresiones ni rupturas, como lo serían los cuerpos e identidades travestis.

Asimismo, otro elemento que destaca y se repite a lo largo de la performance es el quitarse parte de las prendas que visten, siendo este punto entendido como un hecho que revela y exhibe lo que sucede dentro de ellas, ya sean sus sentimientos, pensamientos, o el "implícito" propósito de maquillar. Lo anterior, dado que una vez que ocurre este cambio, también lo hacen los planteamientos de las protagonistas, tomando un giro subversivo e incendiario que apela a la denuncia, la funa y la incomodidad. El acto de desvestirse, puede ser análogo al despojo de ideas, patrones y estigmas heteronormativos y violentos, que incluso han pasado a formar parte de sus vidas e historias, tanto así que influyen en sus modos de ser y percibirse.

En el tercer acto se funden o mezclan ambas personajes y lo que representan, hablando en plural, en pos de comunicar de manera agónica lo que sienten después de estar muertas, cuestión que se simboliza con el derramamiento de la sangre por la calle, simbolizada a partir de pintura roja en botellas. Aquí, el acto de sacarse prendas ya no tiene el mismo significado, sino que continúa con esta idea de "desnudarse" y mostrar que lo que dicen es lo que son y sienten. En este acto final, es donde confluye toda la intensidad de la performance, puesto que se interpreta que ambas protagonistas están hablando desde la muerte, por lo que se refieren en tiempo pasado. Sus diálogos y razones para maquillarse se entremezclan: se maquillaban para sentirse bonitas, pero también para denunciar, para dejar de pertenecer, pero también para pertenecer. Ambas protagonistas llevan el mínimo de ropa y se arrastran, dejando entrever la agonía que llevaban dentro, y que ahora ya no es necesaria ocultar.

Finalmente, el recorrido que hacen las protagonistas es entendido como una analogía a las etapas y experiencias que cruzan la existencia de los cuerpos travestis, que va desde el inicio de la vida travesti hasta su final, y que permite entender que no necesariamente hay una sucesión de hechos lineales durante su desarrollo, ni una limitación en la identidad, es decir, que se deba elegir entre una forma de ser u otra, sino que se puede ser y actuar de incontables maneras, pero que el final será el mismo: cualquier existencia travesti tendrá el mismo destino ingrato.

\section{Expresiones y señales de Apartheid Ocupacional}

A lo largo de toda la performance es posible presenciar elementos que refieren a una situación de apartheid ocupacional, así como a diversas formas de violencias (estructural, social, estatal, física, entre otras). Estas se perciben desde un nivel tanto material como literal, haciendo uso de la burla, la ironía o la rabia.

La primera manifestación de violencia ocurre al comienzo de la performance con una de las protagonistas refiriéndose a la otra, "iquién eres tú? además de un pobre maricón pintado", donde es posible divisar una postura denostativa en torno a su identidad. La alusión a la categoría de "pobre", supone de forma inmediata una inferiorización a partir de la lástima, asimismo, se agrega el término "maricón”, que bajo este contexto no tiene un uso político, sino una connotación ofensiva y de odio que intenta negar e invisibilizar su 
identidad disidente, más aún cuando estos insultos son referidos en masculino. Además, las artistas dan un uso político a aquella comprensión de la heteronorma binaria que asume que el ser travesti radica en "estar pintado", negando la existencia travesti, como una identidad social legítima y sujeta de derechos humanos. En esta misma línea, la frase "obedece y ponte a reproducir" alude a la violencia referida al mandato de reproducción sobre los cuerpos femeninos, cuya improbabilidad en las personas travestis no gestantes conduce a su patologización, fetichización y discriminación a partir de una normatividad esencializada en la biología.

Este mandato no es casualidad, pues tal como sostiene Federici (2018) el trabajo reproductivo constituye un pilar fundamental en la organización capitalista, y por tanto, del trabajo productivo, dado que quienes ofrecen su fuerza de trabajo en los trabajos productivos/remunerados son cuidades, educades, alimentades, satisfeches y servides por quienes ejercen el trabajo de reproducción social ${ }^{8}$. Se puede decir entonces, que este último es esencial -lo que no quiere decir valorado- para el capitalismo, y por ende, aquellos cuerpos que no desempeñan este papel en el sistema son prescindibles, es más, podrían suponer una carga que mantener, ya que no son parte de la fuerza de trabajo remunerable 9 ni son parte de los circuitos formales de producción de esta.

Lo anterior, es en parte la causa y al mismo tiempo la consecuencia de que los cuerpos travestis estén sujetos a una violenta marginación social y económica (Pierce, 2020), y es que además de no cumplir con las expectativas que demandan las normas de género, tampoco cumplirían con lo que imponen las normas capitalistas, entendiendo que ambos sistemas están estrechamente relacionados, pues reproducen el mismo fundamento dicotómico que la matriz binaria, dando espacio a quienes reproducen y a quienes producen, y el resto simplemente no pertenece y es excluido.

Un segundo elemento llamativo, es que a lo largo de la performance ambas protagonistas hacen uso de frases peyorativas, aludiendo al desprecio, al asco y a la indiferencia. Dicho uso puede estar asociado a la visión social y negativa que se tiene en torno a los cuerpos travestis y de lo común de aquellos dichos como una forma de ofensa. Entre estas destaca el uso de “ieres fea!”, “'hija de perra!”, “puta sidosa!” y “iestás enferma!”. Estas guardan relación con un rechazo a sus corporalidades, a su recurrente asociación a la prostitución, a los prejuicios en torno a las Infecciones de Transmisión Sexual (ITS), además de la patologización de cualquier "desviación" a la norma, en este caso, aquella que socialmente les recuerda que sus cuerpos no son coincidentes con los mandatos de género. Dichas formas de violencia afectan el cotidiano de las personas travestis, y son expresadas en planteamientos como, "estoy enferma de criminalizada, enferma de censurada". Todo lo anterior responde a un tipo de violencia estructural, interiorizada e incluso normalizada en las sociedades, que está asociada a las normativas, idiosincrasias y comprensiones existentes que recaen en estos actos políticos. Así, se reproduce un Apartheid Ocupacional en este grupo, que responde a las limitaciones y reproducciones de violencia, reafirmando la idea de que existen categorías de personas que merecen e importan más que otras, siendo las personas travestis quienes reciben estas agresiones y discriminaciones de acuerdo a su posición respecto de la matriz heterosexual y binaria.

\footnotetext{
${ }^{8}$ La autora habla de mujeres, pero para fines de este artículo se referirá a cuerpos reproductivos, ya que, se sostiene que no todas las mujeres tienen cuerpos gestantes y capaces de reproducirse.

${ }^{9}$ Se dice de quienes sostienen la producción del capital, quienes trabajan para ganarse el salario, y quienes a su vez son sostenides y producides por los cuerpos reproductivos.
} 
Otro aspecto a mencionar es el uso de botellas que en su interior contenían un líquido rojo oscuro, que se puede asociar al color de la sangre, y que tras un corte en la presentación es derramada al piso por una de ellas, significando un cambio de escena y de los tiempos verbales implementados, puesto que desde aquí las protagonistas comienzan a hablar en pasado. Dicha escena puede ser asociada a un asesinato causado principalmente por actos violentos, pero no tan solo de una forma directa, sino producto de aquella violencia simbólica y estructural (Buriticá, 2013) que deja como consecuencia una falta de acceso a derechos esenciales como trabajo, educación, salud, entre otros. Esto último es referido por Romero (Fornaro \& Ludueña, 2020) como travesticidios sociales que culminan con la vida de muchas, ya sea en hospitales, desempleadas o abandonadas por el Estado. En la realidad, esto se ve manifestado en las altas cifras de travesticidios, y por consiguiente, la corta esperanza de vida, que en países como Brasil alcanza los 35 años (Instituto Brasileiro de Geografia e Estatística, 2019) y que en el resto de los países latinoamericanos alcanza una expectativa similar.

Además de lo referido anteriormente, las protagonistas agregan "¿quién eres tú? además de una clausura democrática para el Estado" haciendo alusión al permanente e histórico abandono que este ha tenido con la comunidad travesti/trans. Si bien en Chile se han promulgado leyes (Ley Zamudio, Ley Contra la Tortura, Ley de Identidad de Género) que pretenden sancionar los actos discriminatorios y de violencia de las que son víctimas, el respaldo legal y la protección de los derechos de este colectivo continúa siendo insuficiente. Aún existen muchos vacíos, entre ellos y quizás el más importante, es el no reconocimiento de la identidad travesti, lo que produce una serie de vulneraciones, quedando marginadas de sus derechos fundamentales y al acceso a servicios primordiales. En este sentido, es preciso señalar que la identidad travesti a pesar de constituirse como contra-normativa, al mismo tiempo demanda garantías estatales, que les permita tener una vida vivible.

Sobre esto, es preciso analizar el uso de una réplica de la constitución chilena de 1980 durante la performance, manchada con tintura roja similar a la sangre. Desde aquí, se puede apelar a su promulgación en plena dictadura militar, periodo caracterizado por un proceso antidemocrático, vano de pluralismo y participación (Viera, 2011), en un contexto de impunidad y violación a los derechos humanos, que no tan solo fue evidente durante la dictadura militar, sino también en el Chile actual, aquel marcado por la represión, la desigualdad y la violencia sistemática (Muñoz, 2020). Si bien, las corporalidades e identidades travestis nunca han tenido reconocimiento y aceptación social ni estatal, con el inicio de la dictadura militar esta situación se agudizó, siendo la comunidad travesti la minoría sexual más violentada, ultrajada, perseguida e incluso torturada por las fuerzas militares (Zambrano, 2020). Lo expuesto se condice con la frase "iel eje del mal es heterosexual!', que permite relacionar cómo el sistema favorece la violencia y discriminación que viven las disidencias sexuales y de género, cómo este sistema de políticas neoliberales están estrictamente asociadas al "régimen heterosexual" y la "colonización patriarcal" (frases escritas en las espaldas de las protagonistas), y cómo entre sus productos se encuentran las políticas socialistas y neoliberales que no velan ni por los derechos de las personas travestis, ni por su seguridad, representación, visibilización, respeto, etcétera.

\section{Señales de subversión al Apartheid Ocupacional}

Se puede evidenciar que el desarrollo de la performance implica una forma de respuesta y denuncia ante la situación de Apartheid Ocupacional referida. Tal como menciona 
Pérez (2013), la performance permite dilucidar una intención artística e ideológica, que en este caso, se desarrolla en un escenario pandémico y de fuerte represión social, invitando al cuestionamiento del proceso sociopolítico que da paso al plebiscito del año 2020, donde la visibilización y la acción de las identidades contra-normativas se vuelve una forma de expresión, lucha y resistencia. Lo anterior resulta relevante para comprender la potencialidad política no sólo de la performance en su conjunto, sino también de todos los elementos que involucra el mensaje y la propuesta artística. Así, la performance carga con una diversidad de significados que denuncian y deforman la realidad social para posicionar las demandas desde una irrealidad propia (Butler, 2004), dentro de la cual el acto de maquillarse irrumpe, combate, encarna, disputa, dignifica, etc.

Es fundamental no olvidar la potencialidad subversiva, artística y política propia de la identidad travesti, la cual es corporizada como tal en una acción de oposición e inconformismo con la norma y la institucionalidad (Kulawik, 2008). Organa Feminazi expone: "Yo me maquillo, ante todo para festejar, me maquillo para callejear, me maquillo para seducir, (...) me maquillo para putear", así las artistas significan el maquillarse como una acción de incomodar a la norma, pero también de representar una configuración alternativa a la hegemónica. Asimismo, al referirse al callejear y putear es importante comprender las relaciones de clase y cultura que resultan constitutivas y distintivas en la identidad travesti, que trascienden desde la resonancia popular (Pierce, 2020).

En la performance se demuestra que tanto el género como la identidad sexual son una ilusión, una máscara, que sólo es consistente según la cantidad de veces que es repetida por parte de la sociedad (Maureira, 2009), y por lo tanto, da cuenta de su efecto al naturalizarse en torno a un cuerpo (Butler, 2007). Cuando Anna dice "me maquillo porque yo soy la enfermedad" se puede interpretar como un discurso que ha sido repetido constantemente, en donde se identifican los cuerpos fuera de la normativa que se enferma en el acto de subvertirla. En este sentido, Butler (2007) señala que el afán de desnaturalizar el género guarda relación con afrontar las violencias sobre las corporalidades ideales del sexo y las concepciones dominantes en torno a la heterosexualidad. Es aquí donde lo "monstruoso" adquiere protagonismo como una forma de rebelión ante los diferentes mandatos que se imponen sobre sus cuerpos, las artistas toman su identidad, deseos y corporalidad y se fortalecen a través de ella. Anna declara: "Yo me maquillo para sentirme bien, me maquillo para gustarme, me maquillo para sentirme rica, me maquillo para no ser un desastre". Es entonces que, la posibilidad de amar lo que les ha sido negado, se constituye como una forma de subversión frente al mismo.

Se observa también que la performance devela permanentemente los modelos de dominación, por ejemplo, cuando las artistas escriben sobre sus cuerpos "régimen heterosexual" y "colonización patriarcal", y cuando verbalizan los efectos de estas matrices en sus vidas. De la misma manera, se vislumbra en el discurso la idea y acción de transformación, que más concretamente, identifica el maquillarse como una actividad e intencionalidad política que permite subvertir las condiciones que producen estas macroestructuras. Así, maquillarse respalda y otorga seguridad a la identidad, concede sentido y significado en la oposición a la norma, y como señalan las artistas, permite combatir y resistir. Lo anterior reafirma las propuestas de Pardo \& Silva (2015), quienes describen esta potencialidad de las ocupaciones y la cotidianidad de incidir en lo social. Como refiere Van der Fijk, lo político se expresa en todos los aspectos de la vida (Kronenberg \& Pollard, 2006). 
La performance como forma de subversión puede ser leída desde la comprensión de las ocupaciones colectivas, las que desde la mirada de Palacios (2013), son aquellas ocupaciones que tienen una potencialidad de cohesión, de bienestar y de dar sentido de comunidad, un sentido de pertenencia e identidad social, y en donde el territorio alude a la idea de colectividad (Palacios, 2017). Es por ello, que esta ocupación en particular no sólo tiene un sentido de denuncia y subversión, sino que también de unión y creación con la comunidad, y de favorecer un espacio de colectividad en donde se denuncien estas temáticas para cambiar los significados históricamente construidos. El uso del espacio en la performance constituye una acción de apropiación del territorio, y a la vez, interpelación al encuentro con otres, más aún en un escenario pandémico y de restricción. Lo anterior queda patente en el momento en que las performistas despliegan el "escenario" en la calle, adueñándose del espacio que en el día a día constituye un lugar de tránsito. En este caso particular, la performance se desarrolla en Plaza el Descanso, en Valparaíso, sitio icónico de acción colectiva, disidente y popular. Este espacio está cargado de códigos propios que hacen a las artistas poseedoras de él, es el lugar donde se teje la cotidianidad, en donde se está exponiendo, denunciando y agitando contra aquello que dicta la norma.

Se destaca además, el uso del lenguaje durante la performance, como una forma de visibilizar la realidad que les rodea haciendo patentes las vivencias de las personas travestis, y exponiendo entidades y fenómenos, muchas veces ocultos en la vida cotidiana (Sánchez, 2014), que representan una diversidad de significados únicos, y posibilitan la producción de nuevos imaginarios. En la perfomance de Anna Balmánica y Organa Feminazi se utiliza la reiteración para enfatizar la acción política del uso del maquillar, así como también, se incluye el lenguaje del insulto a la identidad travesti, que al ser utilizados por estas mismas constituye una forma de denuncia y representación de una identidad popular y subversiva, una reivindicación de las comunidades disidentes, a través de la apropiación del insulto degradante y posterior expropiación del mismo, que consigue recrear una identidad particular y reafirmar que aquella distinción sexual es un derecho humano (Fonseca \& Quintero, 2009). Del mismo modo, el guión de las performistas recurre de forma explícita a la funa y denuncia de ciertos nombres e instituciones tales como "Nelson Mery" (responsable de torturas y detenciones durante la dictadura militar), "Liceo Carmela Carvajal", "Liceo $\mathrm{N}^{\circ} 1$ " y "Liceo Maya Espinoza" ${ }^{10}$, lo que se constituye como una forma de acción ante las situaciones de violencia e injusticia.

Las protagonistas manifiestan una fuerte crítica a la forma de política que existe en el país desde hace varias décadas, respecto de su relación con las disidencias sexuales y de género, en donde tensionan el rol del socialismo post-dictadura inmediata y del socialismo actual en democracia, infiriendo que este jamás se ha preocupado por incluir a las personas travestis ni sus demandas. Cuando una de ellas exclama “ $A$ Ahh, pero en el 96', el socialismo quiso ser homosexual", y luego “iquién nos ofrecerá el mejor pacto neoliberal?", se entiende la comparación que hace con las campañas políticas, cuando en 1996 el candidato presidencial Eduardo Frei Ruiz-Tagle incluyó en sus propuestas a la comunidad homosexual, para luego no cumplir con sus promesas de campaña durante su gobierno; por otro lado en el 2019, luego de la Revuelta del 18 de octubre, la bancada de derecha junto a parlamentaries de

\footnotetext{
${ }^{10}$ Estos establecimientos educaciones son señalados para dar cuenta de la exclusión de personas travestis, transgénero y transexuales en su interior. Estos Liceos, al ser de "mujeres", han constituido una forma de reforzar estereotipos en torno a lo que se entiende por ser "mujer" donde la disidencia no tiene un lugar. Su nombramiento nos invita a pensar sobre las maneras sexistas en que se reproduce y construye el género desde una institucionalidad que invisibiliza a la diversidad desde edades muy tempranas.
} 
izquierda anunciaron el llamado "Acuerdo por la Paz", que nace producto de las demandas del pueblo chileno por una nueva constitución más equitativa e igualitaria (que en su teoría incluiría a la comunidad LGBTIQA $\left.+{ }^{11}\right)$. Esta comparación nos comunica, que a pesar de que las campańas de la izquierda chilena les han incluido en cada elección de las últimas décadas, sus propuestas sobre igualdad de derechos para la comunidad no han evolucionado, ni han dado cobertura a sus demandas, repitiendo el mismo círculo vicioso de prometer con el fin de ganar adeptos, y luego ignorar dichas promesas, fomentando así la rueda de políticas neoliberales. Ante esto, Organa Feminazi, inquiere "¿quién será la más radical?, ¿quién se lucirá con el mejor pacto neoliberal?” y Anna Balmánica exclama "yo no creo en aquello que nunca ha creído en mî", en un sentido irónico, desafiando a aquelles personajes políticos. Al final de la obra, las performistas señalan “Qué somos nosotras? ¿Por qué queremos votar? ¿Qué hace una travesti marcando apruebo sin cuestionar?", denunciando la falta de representatividad, e invitando a cuestionarse votar por una opción que no asegura ser diferente a los anteriores procesos, preguntándose entonces, por qué votar, y por qué incluirse en un proceso social y político que históricamente no las ha considerado.

Finalmente, las claves visuales, textuales, explícitas e implícitas presentes en esta performance permiten su análisis en torno a las críticas y tensiones que se le hacen a estos sistemas normativos que son la heterosexualidad, el patriarcado y el neoliberalismo, y que de alguna forma permiten entender las conexiones que existen entre ellos, en cómo son productoras de la exclusión y vulneración que viven no sólo las personas travestis, sino las disidencias sexuales y de género en general, y en cómo el "mal" se acuna y desarrolla en estos sistemas a expensas de este grupo de personas, que ahora alzan la voz y denuncian, reflexionan y cuestionan estas relaciones de poder tan impregnadas en la política chilena.

\section{Conclusiones}

A partir de lo investigado, se puede concluir que las instituciones están regidas bajo un conjunto de ideologías neoliberales, patriarcales, que se vinculan a la matriz heterosexual, etc., las que al apoyarse unas con otras y operar desde un nivel estructural, permiten y avalan las acciones de violencia hacia las diversidades sexo genéricas, acciones que son aún más difíciles de erradicar ante la naturalización de sus bases. Lo anterior, a nivel país, tiene su expresión en el Estado neoliberal, la política chilena y las instituciones religiosas judeocristianas, entre otras, las cuales protegen sus intereses y poderes en base a esta discriminación y vulneración, perpetuando así formas particulares de opresión desde una lógica de dominación (capitalista, colonial, binaria, heteronormada, funcionalista, por mencionar algunas), que configuran a las personas y a las sociedades en base a una jerarquía de poder (Hill-Collins, 1990).

No obstante, a pesar del dominio que tienen los modelos hegemónicos sobre la vida cotidiana de las personas, surgen prácticas subversivas que gestan nuevos idearios disidentes de la normatividad que rige, y que levantan la posibilidad de fisurar un sistema que pareciera ser estático e inamovible. Es así, que se configuran diversas estrategias de resistencia que recorren matices ideológicos y artísticos, estos últimos en particular otorgan un espacio fundamental a la expresión de otras subjetividades, haciendo patente aquello que parece no existir.

Con todo esto, lo travesti, entendido como una identidad política, está producida en una histórica violencia y marginación tanto social como económica. En ella destaca su potencial

\footnotetext{
${ }^{11}$ Esta sigla se emplea para referirse a personas lesbianas, gays, bisexuales, transexuales, travestis, transgénero, intersexuales, queer, asexuales y otras $(+)$.
} 
disruptivo que resiste y excede las normas del sistema sexo-género, al escapar de la composición binaria entre lo femenino/mujer y masculino/hombre y de la sexualidad "normativa", ejerciendo su derecho a proclamar su existencia. Por otro lado, se concluye que la ocupación, y en particular las ocupaciones colectivas, tienen características para ser consideradas también políticas, dada la potencialidad transformadora y su capacidad de incidir en cualquier nivel de organización humana individual o colectiva. Cuando se señala que las ocupaciones colectivas posibilitan espacios de fortalecimiento del sentido de comunidad y de pertenencia, y en donde se puede manifestar en contra de lo que dictan las normas, es que se da paso a los procesos de (re)construcción de subjetividades, nuevas cotidianidades, y de micropolíticas liberadoras y de soberanía, que significan agrietar los sistemas de dominación y forjar un destino con autonomía, autodeterminación y de lucha por el ejercicio de derechos.

Ahora, se entiende lo político de las ocupaciones por esta posibilidad de influenciar en lo social y cultural, y porque permiten la conformación de identidades políticas. En este sentido, la performance busca un cambio en el espacio y en quienes lo transitan. Asimismo, esta también es parte de un intercambio, pues recibe respuestas de quienes convoca otorgando espacios de participación mutua, y de compartir sentidos y sentires. Es posible entonces, concluir y proponer que la performance como tal puede ser considerada una ocupación colectiva y política, que tiene la posibilidad de abrir caminos de emancipación entre tantos territorios invadidos por la vulneración, violencia e indiferencia estructurales.

En relación a lo anterior, se tiene que el AO irrumpe en la cotidianidad de las personas travestis coartando sus derechos en muchas esferas de la vida, incluido el acceso a oportunidades y ocupaciones significativas. Frente a esto, es que la performance concibe una posibilidad de quiebre en las barreras que supone el AO, pues implica necesariamente una resistencia producto de un malestar social, permite transformar condiciones sociales desfavorables, de opresión y vulneración con acciones concretas de la vida cotidiana.

Finalmente, en cuanto a las limitaciones de este estudio, se destaca la escasa cantidad de información e investigaciones sobre la población travesti en Chile, y los registros históricos que existen de las performances que se desarrollaron previamente a la era digital son de difícil acceso, lo que impide contextualizar la situación de forma local. Además, los registros históricos de las performances que existen son de difícil acceso, dado que gran parte de ellas se pueden encontrar únicamente en bases de datos de instituciones que no tienen información de manera pública, quedando este acceso sujeto a la disponibilidad y disposición de quienes poseen los derechos de autoría de las obras.

\section{Referencias}

Aguiar, B., Encina, F., Iturriaga, C., Jiménez, C., Morrison, R., \& Riquelme, E. (2020). Aproximación al transfomismo desde una perspectiva ocupacional y de género. In J. M. Valcuerde, A. Robles, M. A. Salguero \& J. O. Hernández (Eds.), Diversidad Sexual en Iberoamérica. Derechos, Placeres y Vivencias (pp. 373-394). México: UNAM.

Aguilar, M. (2016). Reflexiones conceptuales y metodológicas sobre análisis y producción audiovisual en sociología. Revista de Metodología de Ciencias Sociales, (35), 153-174. http://dx.doi.org/10.5944/empiria.35.2016.17172.

Argentina. (2014, 9 de maio). Ley 26.743. Establécese el derecho a la identidad de género de las personas. Boletin Oficial de la República Argentina, Buenos Aires.

Avillo, C., Barrientos, B., Fernández, J., Matus, C., Olivares, C., \& Morrison, R. (2015). Contexto y Elecciones Ocupacionales de adolescentes que se identifican como homosexuales. Revista Chilena de Terapia Ocupacional, 15(2), 1-19. http://dx.doi.org/10.5354/0717-5346.2015.38157. 
Balmánica, A. (2020). En el pandémico contexto del aniversario de la revuelta popular del 18 de octubre, las travestis volvemos a tomarnos las calles. Instagram. Recuperado el 5 de agosto de 2021, de https://www.instagram.com/p/CGYr0CmHWEe/

Barbieri, T. (1993). Sobre la categoría género: una introducción teórico-metodológica. Debates en Sociología, (18), 145-169.

Bonassi, B., Santos, M., Toneil, M., \& Queiroz, M. (2015). Vulnerabilidades mapeadas, violências localizadas: experiências de pessoas travestis e transexuais no Brasil. Quaderns de Psicologia, 17(3), 83-98.

Bonilla, M., \& López, A. (2016). Ejemplificación del proceso metodológico de la teoría fundamentada. Cinta de Moebio, (57), 305-315. http://dx.doi.org/10.4067/S0717-554X2016000300006.

Boy, M., \& Rodríguez, M. (2019). Acceso a la salud de travestis y trans: espacios cis-heteronormados y cuerpos (im)pacientes: Noroeste del Gran Buenos Aires, provincia de Buenos Aires, 2016-2018. In Anales de $4^{\circ}$ Seminario Latinoamericano de Geografía, Género y Sexualidades. Buenos Aires: UNCPBA.

Braga, I. F., Melo, K. M. M., Monzeli, G. A., Leite Junior, J. D., Farias, M. N., \& Correia, R. L. (2020). Crise da democracia brasileira e o cotidiano de pessoas dissidentes de gêneros e sexualidades: reflexóes baseadas na terapia ocupacional social. Cadernos Brasileiros de Terapia Ocupacional, 28(2), 693-705. http://dx.doi.org/10.4322/2526-8910.ctoarf1958.

Buriticá, I. (2013). Travesti: la construcción de la identidad individual y colectiva desde el cuerpo y el ejercicio de la prostitución. La Manzana de la Discordia, 8(2), 71-86.

http://dx.doi.org/10.25100/lamanzanadeladiscordia.v8i2.1541.

Butler, J. (2002). Cuerpos que importan: sobre los limites materiales y discursivos del "sexo". Argentina: Paidós

Butler, J. (2004). Deshacer el género. Argentina: Paidós.

Butler, J. (2007). El género en disputa: el feminismo y la subversión de identidad. Argentina: Paidós.

Butler, J. (2017). Cuerpos aliados y lucha politica: hacia una teoría performativa de la asamblea. Argentina: Paidós.

Carrasco, D. (2015). Las travestis no necesitan interlocutores, por que en cualquier momento te pueden explotar en la cara, o cómprame por kilo de huesos. Una presentación para "Cuerpos para odiar". Recuperado el 5 de agosto de 2021, de https://es.scribd.com/document/273834255/Las-Travestis-No-Necesitan-Interlocutores-PorQue-en-Cualquier-Momento-Te-Pueden-Explotar-en-La-Cara-o-Comprame-Por-Kilo-de-Huesos

Castillo, S., \& Mayo, S. (2019). El lenguaje inclusivo como "norma” de empatía e identidad: reflexiones entre docentes y futures profesores. Literatura y Lingüistica, (40), 377-391. http://dx.doi.org/10.29344/0717621x.40.2072.

Cellino, R. (2015). Performance: travestismos y política en las crónicas de Pedro Lemebel. Catedral Tomada. Revista de Critica Literaria Latinoamericana, 3(4), 21-49. http://dx.doi.org/10.5195/ct/2015.83.

Centro de Documentación y Situación Trans en Latinoamérica y el Caribe - CeDoSTALC. (2017). Informe de abusos y violaciones a los derechos humanos de las personas trans en Chile, durante el período 2016/2017. Panamá: CeDoSTALC.

Coñuenao, J. (2019). Las fronteras del género: una nueva instancia para el debate. observatorio de medios y movimientos sociales. Chile: Universidad de la Frontera.

Cortés, L., Polanco, M., Retamal, M., Guerra, K., \& Farfán, S. (2016). Lo performativo en la performance art. Revista Colombiana de las Artes Escénicas, 10, 9-20.

Dulzaides, M., \& Molina, A. (2004). Análisis documental y de información: dos componentes de un mismo proceso. Acimed, 12(2), 1-5.

Duque, C. (2010). Judith Butler y la teoría de la performatividad de género. Revista de Educación y Pensamiento, (17), 85-95.

Federici, S. (2018). El Patriarcado del salario. Críticas feministas al marxismo. Buenos Aires: Tinta Limón.

Fonseca, C., \& Quintero, M. (2009). La Teoría Queer: la de-construcción de las sexualidades periféricas. Sociologica, 24(69), 43-60.

Fornaro, A., \& Ludueña, M. (2020). "Los travesticidios sociales son crimenes de odio”. Presentes. Recuperado el 5 de agosto de 2021, de https://agenciapresentes.org/2020/06/29/los-travesticidios-sociales-son-crimenes-de-odio/ 
Galheigo, S. (2003). O cotidiano na terapia ocupacional: cultura, subjetividade e contexto histórico-social. Revista de Terapia Ocupacional da Universidade de São Paulo, 14(3), 104-109.

Galheigo, S. (2020). Terapia ocupacional, cotidiano e a tessitura da vida: aportes teórico-conceituais para a construção de perspectivas críticas e emancipatórias. Cadernos Brasileiros de Terapia Ocupacional, 28(1), 5-25. http://dx.doi.org/10.4322/2526-8910.ctoao2590.

García, A. (2010). Tacones, siliconas, hormonas. Teoría feminista y experiencias trans en Bogotá (Tesis de magíster). Universidad Nacional de Colombia, Colombia.

Gómez, R. (2013). Emergencia y rebase en la pose y puesta en práctica del cuerpo transformistaltravesti (trans/trave) en Chile (Tesis de magíster). Pontificia Universidad Católica de Chile, Chile.

Gómez-Antilef, B. F., Heise-Barrera, C. A., Muñoz-Vidal, G. A., Velásquez-Bórquez, G. E., VerdugoÁlvarez, V. N., \& Carrasco-Madariaga, J. S. (2020). Construcción de subjetividad trans a partir de la ocupación entendida como hacer, ser y llegar a ser. Cadernos Brasileiros de Terapia Ocupacional, 28(2), 419-435. http://dx.doi.org/10.4322/2526-8910.ctoAO1960.

Guajardo, A. (2012). Enfoque y Praxis en Terapia Ocupacional. Reflexiones desde una perspectiva de la terapia ocupacional crítica. TOG (A Coruña), 9(5), 18-29.

Gutierres, S. M., \& Lordello, S. R. (2020). Promoção da saúde com transexuais e travestis: uma revisão sistemática de literatura. Revista Psicologia Política, 20(47), 165-177.

Haraway, D. (1991). Ciencia, cyborgs y mujeres. La reinvención de la naturaleza. Espanha: Cátedra.

Hill-Collins, P. (1990). Black Feminist Thought: knowledge, consciousness and the politics of empowerment. Londres: Routledge.

Instituto Brasileiro de Geografia e Estatística - IBGE. (2019). Tábua completa de mortalidade para o Brasil 2018: breve análise da evolução da mortalidade no Brasil. Rio de Janeiro: IBGE.

Instituto Nacional de Derechos Humanos - INDH. (2012). Informe sobre Chile-violación a los DDHH de personas transexuales. Recuperado el 5 de agosto de 2021, de https:/www.indh.cl/wpcontent/uploads/2012/03/EPU-OTD.pdf

Kronenberg, F., \& Pollard, N. (2006). Introducción: Un comienzo. In F. Kronenberg, S. S. Algado \& N. Pollard (Eds.), Terapia Ocupacional sin Fronteras (pp. 1-13). Bogotá: Médica Panamericana.

Kulawik, K. (2008). Travestir para reclamar espacios: la simulación sex-/textual de Pedro Lemebel y Francisco Casas en la urbe chilena. Alpha, 26(26), 101-117. http://dx.doi.org/10.4067/S071822012008000100007.

Lamas, M. (2017). Conferencias clave para entender el género por Marta Lamas [Vídeo]. Recuperado el 5 de agosto de 2021, de https://www.youtube.com/watch?v=37P9C2xMfaU

Lefebvre, H. (1991). Critique of everyday life. London: Verso.

Marchant Castilho, J. (2019). Posibles abordajes de terapia ocupacional en la educación sexual de niños, adolescentes y jóvenes lesbianas, gay, bisexuales y transgéneros. Revista Chilena de Terapia Ocupacional, 19(2), 63-72. http://dx.doi.org/10.5354/0719-5346.2019.53411.

Martínez, P. (2006). El método de estudio de caso: estrategia metodológica de la investigación científica. Pensamiento \& Gestión, (20), 165-193.

Maureira, I. (2009). La deconstrucción del nombre propio en la nominación travesti. Alpha, (29), 155-165. http://dx.doi.org/10.4067/S0718-22012009002900011.

Melo, K. M. M. (2016). Terapia Ocupacional Social, pessoas trans e Teoria Queer: (re)pensando concepçóes normativas baseadas no gênero e na sexualidade. Cadernos Brasileiros de Terapia Ocupacional, 24(1), http://dx.doi.org/10.4322/0104-4931.ctoARF0645.

Monzeli, G. (2013). Em casa, na pista ou na escola é tanto babado: espaços de sociabilidade de jovens travestis (Dissertaçao de mestrado). Universidade Federal de São Carlos, São Carlos.

Monzeli, G. A., Ferreira, V. S., \& Lopes, R. E. (2015). Entre proteção, exposição e admissões condicionadas: travestilidades e espaços de sociabilidade. Cadernos de Terapia Ocupacional da UFSCar, 23(3), 451-462. http://dx.doi.org/10.4322/0104-4931.ctoAO0518. 
Moraga, M. (2017). Apartheid Ocupacional de las diversidades trans: Importancia y desafío para la Terapia Ocupacional Social. Polyphōnia, 1(2), 106-118. Recuperado el 5 de agosto de 2021, de https://revista.celei.cl/index.php/PREI/article/view/236

Morrison, R., Araya, L., Palomino, T., \& Graus, J. M. (2019). Situación de las niñas y niños trans en Chile: derechos humanos y políticas públicas. RELIES: Revista del Laboratorio Iberoamericano para el Estudio Sociohistórico de las Sexualidades, (1), 26-48.

Morrison, R., Araya, L., Valle, J. D., Vidal, V., \& Silva, K. (2020). Occupational apartheid and human rights: narratives of Chilean same-sex couples who want to be parents. Journal of Occupational Science, 27(1), 39-53. https://doi.org/10.1080/14427591.2020.1725782.

Movimiento de Integración y Liberación - Movilh Chile. (2021). XIX. Informe Anual de Derechos Humanos de la Diversidad Sexual y de Género en Chile (Hechos 2020). Santiago: Movilh Chile.

Muñoz, G. (2020). Impunidad: El pacto constituyente está manchado con la sangre de nuestros heridos y muertos. Recuperado el 5 de agosto de 2021, de http://www.laizquierdadiario.cl/Impunidad-El-pactoconstituyente-esta-manchado-con-la-sangre-de-nuestros-heridos-y-muertos

Nayar, S., \& Stanley, M. (2014). Qualitative research methodologies for occupational science and therapy. Londres: Routledge.

Neer, A. F. (2017). Travestismo, transexualidad y transgeneridad en los discursos del estado Argentino: Desde los edictos policiales hasta la Ley de Identidad de Género. Buenos Aires: Teseo.

Palacios, M. (2013). Ocupación colectiva, sentido de comunidad y bienestar psicosocial. In Colegio de Terapeutas Ocupacionales de Chile, P. Caro-Vines, R. Morrison \& M. Palacios (Eds.), Cincuenta años de terapia ocupacional en Chile (Tomo I, pp. 143-159). Santiago: Ediciones On Demand.

Palacios, M. (2017). Acerca del sentido de comunidad, ocupaciones colectivas y bienestar/malestar psicosocial. Con jóvenes transgresores de territorios populares (Tesis doctoral). Universitat de Vic, España.

Pardo, M., \& Silva, G. (2015). Dimensión política de las cotidianidades: implicancias del enfoque de género en las intervenciones de terapia ocupacional. In Colegio de Terapeutas Ocupacionales de Chile, P. CaroVines, R. Morrison \& M. Palacios (Eds.), Cincuenta años de terapia ocupacional en Chile (Tomo I, pp. 113-128). Santiago: Ediciones On Demand.

Pérez, A. (2013). Arte y política: nuevas experiencias estéticas y producción de subjetividades. Comunicación y Sociedad, (20), 191-210.

Pérez, G. (1998). Investigación Cualitativa. Retos e interrogantes. Métodos (Tomo I). Madrid: La Muralla.

Pierce, J. (2020). I Monster: Embodying Trans and Travesti Resistance in Latin America. Latin American Research Review, 55(2), 305-321. http://dx.doi.org/10.25222/larr.563.

Polanco, N., \& Morrison, R. (2019). Patriarcado, masculinidad hegemónica y ocupaciones: procesos de perpetuación del sexismo. Revista Argentina de Terapia Ocupacional, 5(1), 75-84. Recuperado el 5 de agosto de 2021, de http://www.revista.terapia-ocupacional.org.ar/RATO/2019jul-ens.pdf

Pollard, N., Kronenberg, F., \& Sakellariou, D. (2008). Occupational apartheid. In N. Pollard, D. Sakellariou \& F. Kronenberg (Eds.), A Political Practice of Occupational Therapy (pp. 55-68). Rio de Janeiro: Elservier.

Presentes LGTB. (2017). Entrevista a la activista trans chilena Claudia Rodríguez [Vídeo]. YouTube. Recuperado el 5 de agosto de 2021, de https://www.youtube.com/watch?v=4SZxlVE7cvs

Radi, B., \& Sardá-Chandiramani, A. (2016). Travesticidioltransfemicidio: Coordenadas para pensar los crimenes de travestis y mujeres trans en Argentina. Colombia: Boletín del Observatorio de Género.

Sánchez, V. (2014). Identidades de Género y Sexuales en el Lenguaje. Lenguaje Políticamente Correcto: Dos Caras de la Moneda. In Anales de 5 a Jornadas de Graduados-Investigadores en Formación. La Plata: Universidad Nacional de La Plata.

Scott, J. (1996). El género: una categoría útil para el análisis histórico. In M. Lamas (Comp.), El Género, la construcción cultural de la diferencia sexual (pp. 265-302). México: PUEG. 
Silva, C., Cardinalli, I., Sanches, M., Zacchi, A., Almeida, A., Ambrosio, L., Taliane de Oliveira, M., \& de Paula, B. (2017). La terapia ocupacional y la cultura: miradas a la transformación social. Revista Chilena de Terapia Ocupacional, 17(1), 105-113. http://dx.doi.org/10.5354/0719-5346.2017.46383.

Tarrés, M. (2013). A propósito de la categoría género: leer a Joan Scott. Estudios Sociológicos, 31(91), 3-26.

Toro, C., \& Carrasco, D. (2019). Anna Balmánica: "Las drag queen estamos mejor posicionadas para transmitir el mensaje de otras problemáticas $L G B T$ ”. Recuperado el 5 de agosto de 2021, de https://www.elmostrador.cl/tv/2019/05/04/anna-balmanica-las-drag-queen-estamos-mejorposicionadas-para-transmitir-el-mensaje-de-otras-problematicas-lgbt/

Torres, M. (2013). Crimen, Pasión y Muerte: una visión geográfica de la experiencia travesti en las cárceles masculinas de Santiago de Chile. Global Journal of Human Social Science Interdisciplinary, 13(3), 7-17. Recuperado el 5 de agosto de 2021, de https://studylib.es/doc/7095426/crimen--pasi\%C3\%B3n-ymuerte--una-visi\%C3\%B3n-geogr\%C3\%A1 fica

Valdés, G. (2016). La codificación Axial, innovación metodológica. Revista Electrónica Científica de Investigación Educativa, 3(1), 497-509. Recuperado el 5 de agosto de 2021, de https://www.rediech.org/ojs/2017/index.php/recie/article/view/244/325

Vidiella, J. (2014). Performatividad y poder. Políticas de representación e identidad: corporización y performance. Revista DCO, (7), 6-8.

Viera, C. (2011). Análisis Crítico de la génesis de la Constitución vigente. Revista de Derechos Fundamentales, (5), 151-171.

Zambrano, A. (2020). Retratos de un travestismo en dictadura. "La manzana de Adán» de Paz Errázuriz y Claudia Donoso. Recuperado el 5 de agosto de 2021, de https://www.revistatransas.com/2020/05/14/retratos-de-untravestismo-en-dictadura-la-manzana-de-adan-de-paz-errazuriz-y-claudia-donoso/

\section{Contribución de los Autores}

Mariana, Camila, Marianne y Sofía realizaron la revisión bibliográfica, seleccionaron el material empírico y realizaron el análisis documental, además, redactaron la primera versión del texto y aprobaron su versión final. Débora y Rodolfo dirigieron la investigación, realizaron aportes teóricos, revisaron y aprobaron la versión final del escrito.

Todos los autores aproban la version final del texto.

\section{Fuente de Financiamiento}

Departamento de Terapia Ocupacional y Ciencia de la Ocupación de la Universidad de Chile.

Débora Grandón-Valenzuela recibe el apoyo de la Agencia

Nacional de Investigación y Desarrollo (ANID),

PFECHA/Doctorado Nacional 2020/21201656.

\section{Autor para la correspondencia}

Marianne Pfeifer Fuentes

e-mail: marianne.pfeifer@ug.uchile.cl

\section{Editor de sección}

Profa. Dra. Daniela Edelvis Testa 